\title{
Purine and monoamine metabolites in cerebrospinal fluid: parallel purinergic and monoaminergic activation in depressive illness?
}

\author{
F NIKLASSON, H ÅGREN, R HÄLLGREN \\ From the Departments of Clinical Chemistry, Psychiatry and Internal Medicine, University Hospital, Uppsala, \\ Sweden
}

SUMMARY In the cerebrospinal fluid of 38 patients with major depressive disorders the purine metabolites hypoxanthine and xanthine were positively correlated to the monoamine metabolites HVA and 5HIAA ( $p<0.0001)$. Hypoxanthine was also positively linked to the noradrenaline metabolite MHPG ( $p<0.005)$. By the use of multiple regression analysis $70 \%$ of the variance in hypoxanthine and $51 \%$ of the variance in xanthine were explained by HVA and 5HIAA. The scored magnitude of memory disturbance during depression was positively correlated to hypoxanthine, xanthine, HVA, and 5HIAA, while the degree of somatic anxiety as well as worrying was or tended to be negatively correlated to the same biochemical markers. The conspicuous relationship observed between purine and monoamine matabolite concentrations in CSF during depressive illness might indicate a parallel purinergic and monoaminergic activation of the brain. The observation that certain isolated depressive symptoms appear to relate to hypoxanthine/xanthine in CSF is consistent with the hypothesis of a central role of purines in behaviour.

Adenosine and its nucleotides are extensively involved in brain metabolism. Accumulated data also indicate that these compounds may act as neurotransmitters or neuromodulators in the central nervous system (for review see Phillis and $\mathrm{Wu}^{1}$ ). Adenine nucleotides are stored within brain cells with ATP being the prevalent form. ${ }^{2}$ During stimulation of central nervous structures in vitro and in vivo adenine nucleotides are released ${ }^{3-6}$ and then rapidly hydrolysed extracellularly to form the purine nucleosides. Further degradation results in hypoxanthine and xanthine; the end products of the purine metabolism, while the absence or very low amounts of xanthine oxidase in the brain ${ }^{7}$ is not consistent with the formation of urate. Recently we have noted that the hypoxanthine and xanthine concentrations in the cerebrospinal fluid of patients with major depressive disorders were strongly related to certain psychiatric variables including suicidal tendency, worrying and memory disturbance ( ̊̊gren, Niklasson, Hällgren, to

Address for reprint requests: $\mathbf{R}$ Hällgren, Department of Internal Medicine, University Hospital, S-75014, Uppsala, Sweden

Received 12 August 1982 and in revised form 17 October 1982 Accepted 29 October 1982 be published). Since similar behavioural changes have previously been reported to relate to monoamine metabolites in $\mathrm{CSF}^{89}$ we suspected that adenine nucleotides may be released in parallel with monoamine transmitters during stimulation of the brain. We have therefore searched for a relationship between the CSF levels of hypoxanthine-xanthine and monoamine metabolites.

\section{Patients and methods}

Thirty-eight patients were diagnosed as suffering from major depressive disorder according to Research Diagnostic Criteria. Structured interviews were conducted with the SADS (Schedule for Affective Disorders and Schizophrenia $\left.{ }^{10}\right)$. Twenty-five were unipolars and 13 bipolars. Twenty were females, mean age 41 (range 20-66) years, and 18 males, mean age 42 (range 22-57) years. No antidepressant or neuroleptic medication was allowed during a ten-day period before tests. Twelve patients were on regular benzodiazepine regimen, 16 used it irregularly during the investigation period, and 10 had no contact with this class of drugs. Only two patients had been treated with lithium at any time. The cerebrospinal fluid (CSF) was collected by lumbar puncture performed in a standardised manner as described previously. "A fixed volume of $13 \mathrm{ml}$ was collected. The specimens were immediately frozen and 

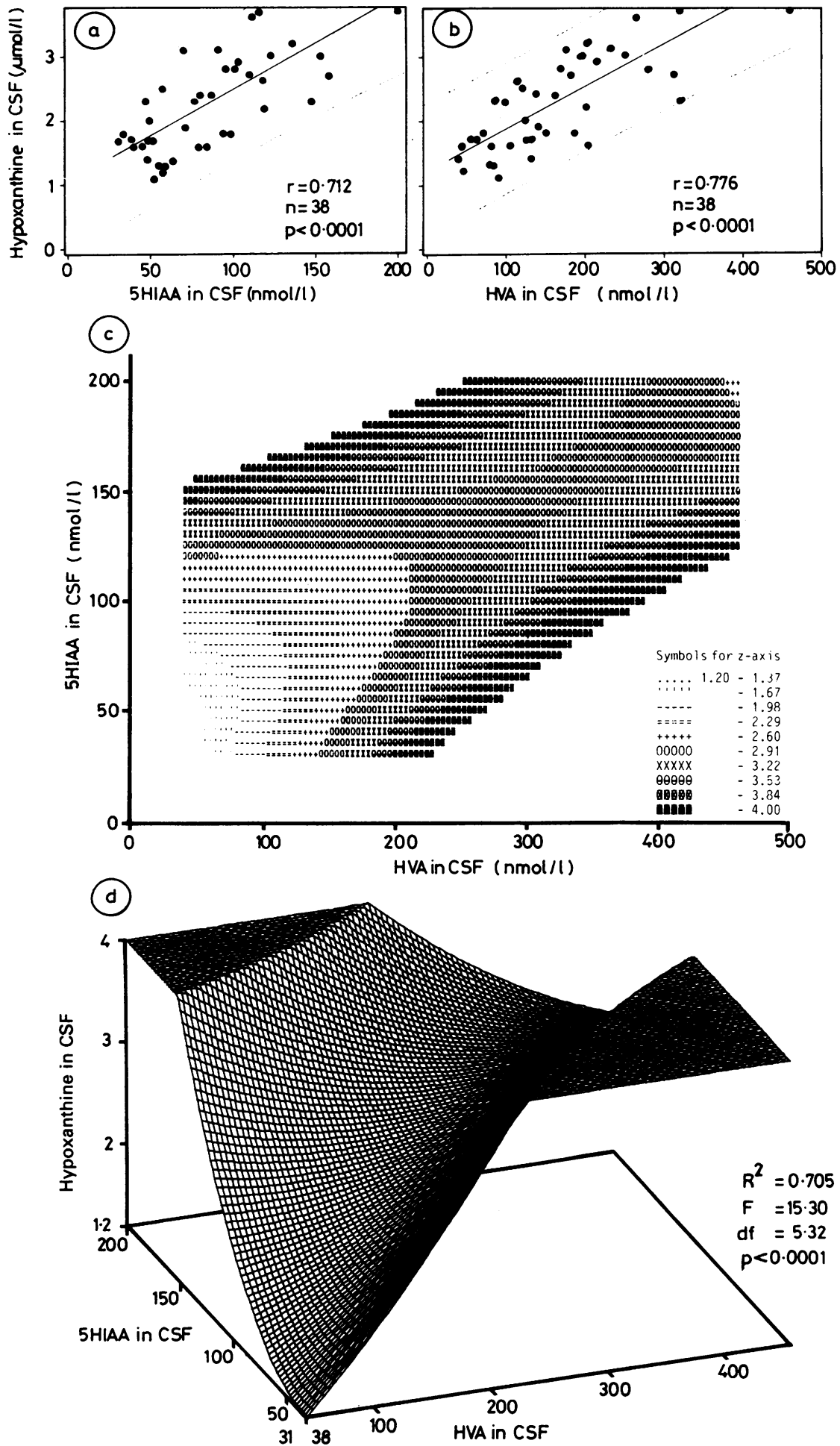

Fig 1 Trivariate correlations between hypoxanthine in CSF on the one hand, and homovanillic acid (HVA) and 5-hydroxyindoleacetic acid (5HIAA) in CSF on the other. Plots $A$ to $B$ show univariate regressions between hypoxanthine and $5 H I A A(A)$ and $H V A(B)$, with $95 \%$ confidence limits drawn. Plots $C$ and $D$ are three-dimensional representations of curvilinear and interactional regression surfaces; hypoxanthine is the $z$ axis, HVA $x$ axis, and SHIAA y axis. See text for details on statistics. 

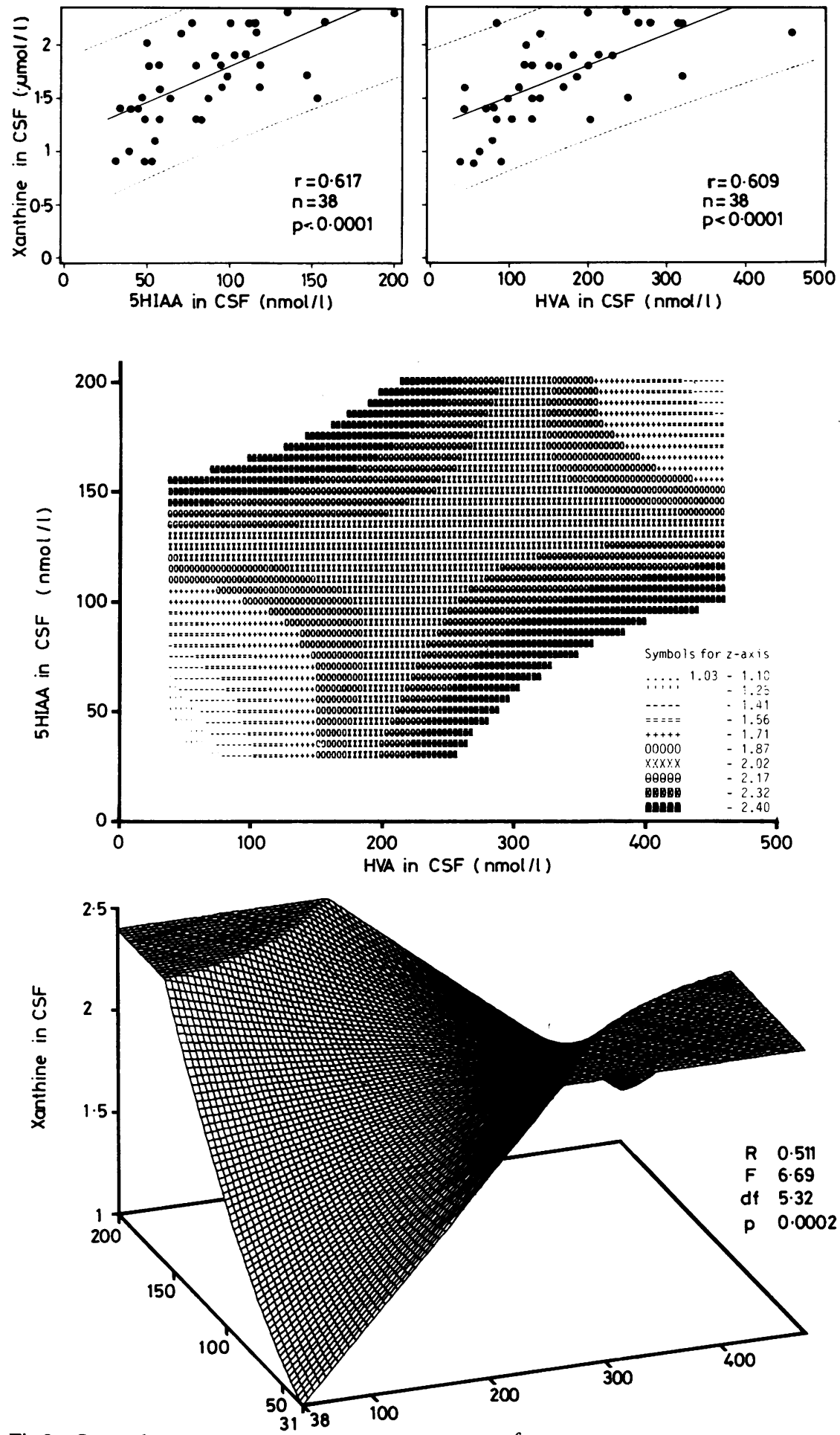

Fig 2 Same plots as in fig 1, but with xanthine in CSF in focus. 
Table Univariate correlations between selected depressive symptoms and hypoxanthine, xanthine, $H V A$, and $5 H I A A$ in the CSF. Pearson rare shown in regular face, and Spearman $r_{s}$ in italics. $N=38$

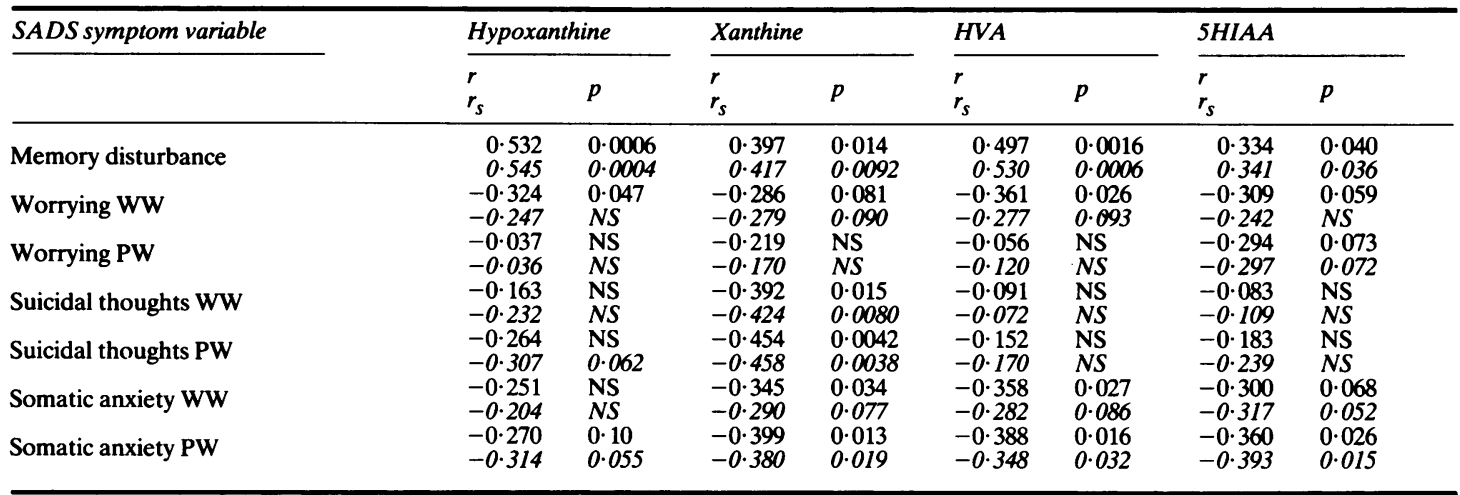

Notes:

NS $=$ Non-significance and non-trend, $>0 \cdot 10$.

WW $=$ Worst Week of Present or Recent Depressive Episode.

PW $=$ Past Week Before Investigation.

analysis of monoamine metabolites were carried out within two weeks by Professor Lars Svennerholm in Gothenburg according to a procedure described. ${ }^{12}$ The monoamine metabolites analysed were homovanillic acid (HVA), 5hydroxy-indoleacetic acid (5HIAA) and 3-methoxy-4hydroxyphenyl glycol (MHPG). CSF specimens for hypoxanthine and xanthine analyses were stored at $-70^{\circ} \mathrm{C}$ until analysed in sequence by means of a reversed phase high performance liquid chromatography equipment as reported elsewhere (Hällgren $R$, Niklasson $F$, Terént $A$, Åkerblom $\AA$, Widerlöv E. Oxypurines in cerebrospinal fluid as indices of disturbed brain metabolism. To be published).

All data were stored on computer files and processed by an IBM 4341 main-frame computer. SAS (Statistical Analysis System Inc) procedures like Correlation, General Linear Models, Plot and Graph Options were employed.

\section{Results}

Strong positive correlations emerged between the purine metabolites and HVA and 5HIAA in CSF (hypoxanthine-HVA, Pearson $r=0.776, p<0.0001$; hypoxanthine-5HIAA, $\mathrm{r}=0.712, \mathrm{p}<0.0001$; xanthine-HVA, $r=0.609, p=0.0001$; xanthine5HIAA, $\mathrm{r}=0.617, \mathrm{p}=0.0001 ; \mathrm{n}=38$ for all). The regression lines for these correlations are given in figs 1 and 2, Plots A and B. The $95 \%$ confidence limits drawn show that practically no value lies outside this interval. There was a weaker, but still significant correlation between hypoxanthine and the noradrenaline metabolite MHPG in CSF $(r=0.448$, $\mathrm{n}=38, \mathrm{p}=0.005$ ); no link was found between xanthine and MHPG $(r=0 \cdot 300, p=0 \cdot 07)$.

If each purine metabolite was correlated with HVA and 5HIAA in a multiple regression analysis with squared values of HVA and 5HIAA and an interaction term, HVA multiplied with 5HIAA, added as three other predictor variables, the results were quite distinct. Hypoxanthine correlated with this quadratic and interactional equation to the effect that $70 \%$ of the variance in hypoxanthine was explained by the predictors present $\left(R^{2}=0.705 ; \mathrm{F}=\right.$ $15 \cdot 30 ; \mathrm{df}=5 \cdot 32 ; \mathrm{p}<0.0001)$. With only the simple variables HVA and 5HIAA in the equation, variance explained was only $63 \%\left(R^{2}=0 \cdot 636 ; F=30 \cdot 64 ; \mathrm{df}=\right.$ $2 \cdot 35 ; \mathrm{p}=0 \cdot 0001)$. Thus, the quadratic terms and the interaction added $7 \%$. Similarly, the variance in xanthine was considerably explained by the same five predictors to $51 \%\left(\mathrm{R}^{2}=0.511 ; \mathrm{F}=6.69 ; \mathrm{df}=5.32\right.$, $\mathrm{p}=0.0002)$. With only two simple variables, the degree of variance explained decreased to $42 \%\left(R^{2}=\right.$ $0.426 ; \mathrm{F}=12.96 ; \mathrm{df}=2.35 ; \mathrm{p}=0.0001$ ); thus, curvilinearity and interaction added $9 \%$. These complex equations are visualised in three dimensions in figs 1 and 2, plots $C$ and $D$. In both methods of 3-D depiction utilised, hypoxanthine and xanthine, respectively, are at the $z$ axes. The "wing flaps" in figs 1 and 2, plots D, are computational artifacts.

Another way of demonstrating connections between purine and monoamine metabolites in CSF is by examining behavioural correlates shared in common. In another report, we have tried to show that, for example, memory disturbance during a depression is linked with higher levels of hypoxanthine in CSF, and worrying, suicidal tendencies and somatic anxiety to be connected with lower levels of xanthine (Ågren et al, to be published). This selection of depressive symptoms resembles quite closely that found in relation with HVA and 5HIAA in an earlier study. ${ }^{9}$ In the table, four symptoms (scored from the Schedule for Affec- 
tive Disorders and Schizophrenia, $\operatorname{SADS}^{10}$ ), are shown univariately correlated with hypoxanthine, xanthine, HVA, and 5HIAA in CSF, using both Pearson's product-moment $r$ and Spearman's rankcorrelation $r_{s}$ (for Worst Week of present or recent depressive episode, and for Past Week before investigation). It should be noted that correlations between suicidal symptomatology and monoamine matabolites have been found in studies using other scores of suicidality. ${ }^{89}$

\section{Discussion}

Regulation of the turnover of monoamines in the central nervous system has been the subject of extensive studies. Experiments using receptor blocking agents have demonstrated the regulatory role of certain receptors on monoamine synthesis and utilisation. ${ }^{13}$ However, the molecular mechanisms by which such receptors regulate the turnover of the monoamines are largely unknown. The present study demonstrating a conspicuous relationship between purine and monoamine metabolites in CSF of patients with depression may be considered as a clinical support for the hypothesis that adenosine and related nucleotides modulate the release of neurotransmitters including monoamines.

Attention to the role of nucleotides in neuronal function (other than cyclic AMP) has been steadily increasing during recent years and has been the subject of a recent review. ${ }^{1}$ Several studies have demonstrated an extracellular release of purines from brain tissues. ${ }^{34617}$ The correlation observed in CSF between hypoxanthine and xanthine and monoamine metabolites might possibly reflect a parallel release of purines and monoamines from synaptic vesicles. The issue of such a co-release has some support by the finding that ATP is stored in catecholamine storage granules of the splenic nerve trunk ${ }^{14}$ and in cholinergic vesicles. ${ }^{1516}$ Stimulation leads to a depletion of vesicle contents of both acetylcholine and ATP. However, there exists no direct evidence of a co-storage of ATP with monoamines in the CNS.

Another possible explanation of the relationship between monoamine and purine degradation products is based upon the presynaptic site of action of adenosine and its nucleotides with inhibition of neurotransmitter release, including that of dopamine and serotonin. Studies on peripheral neurons indicate that such mechanisms may be involved. ${ }^{18-20}$ If the same holds true in central neurons an increased presynaptic neuronal activation would induce an enhanced release of adenine-related compounds ${ }^{1}$ thereby inhibiting a further synaptic transmitter release. Such a negative feed-back regulation would be reflected by a balanced synthesis and release of purines and monoamines and explain the present finding. A corollary of the above arguments would be that the purine end products detectable in CSF originate to a considerable extent from adenine nucleotides released during neuronal activity.

During cerebral ischaemia the intracellular concentrations of adenine and guanine di- and trinucleotide rapidly fall. ${ }^{2122}$ In parallel with these events, the corresponding monophosphonucleotides increase in cerebral tissue. These consequences of decreased oxygen supply are reflected in increased hypoxanthine and xanthine concentrations in CSF (Hällgren et al, to be published). In this situation, the increased release of oxypurines is supposed to occur from an intracellular metabolic pool, rather than from a presynaptic vesicle pool. The purine metabolites in CSF may thus also reflect the brain metabolism and not only a balanced release from nervous structures. If so, the relationship found between hypoxanthine-xanthine and monoamine metabolites in CSF might reflect the influence of the brain metabolic rate on the monoamine synthesis, or, alternatively, the regulatory role of monoamines on the brain metabolism.

Evidence exists for behavioural changes due to nucleotide action. There are a number of reports describing sedative and anticonvulsant effects of adenine, adenosine and various adenosine analogues in experimental animals. ${ }^{23-26}$ The Lesch-Nyhan syndrome, an inherited disorder of purine metabolism with increased concentrations of urate and oxypurines in blood and CSF is characterised by mental retardation, psychomotor retardation and self-mutilation. ${ }^{27}$ It has also been suggested that some actions common to several groups of psychotherapeutic agents may be exerted by effects on adenosine and ATP in central neurons. ${ }^{1}$ Like previous investigators, we found that affective symptoms such as suicidal measures and memory disturbance were connected with different CSF concentrations of 5HIAA and HVA. ${ }^{8}$ These symptoms were correlated in our patients with CSF levels of hypoxanthine and/or xanthine as well. Thus, certain behavioural variables appear to be least common denominators behind the purinergic and monoaminergic metabolism as reflected in CSF during depressive illness.

This project was in part supported by the Swedish Medical Research Council (grant K82-21P-6355-01) and the Medical Faculty at Uppsala University. Analytical assistance from Åke Åkerblom is acknowledged.

\section{References}

${ }^{1}$ Phillis JW, Wu PH. The role of adenosine and its nucleotides in central synaptic transmission. Prog Neurobiol 1981;16:187-239. 
${ }^{2}$ Santos JN, Hempstead KW, Kopp LE, Miech RP. Nucleotide metabolism in rat brain. $J$ Neurochem 1968;15:367-76.

${ }^{3}$ Pull I, Mcllwain H. Adenine derivatives as neurohumoral agents in the brain. The quantities liberated on excitation of superfused cerebral tissues. Biochem $J$ 1972;130:975-81.

${ }^{4}$ Schubert P, Rose G, Lee K, Lynch G, Kreutzberg GW. Axonal release and transfer of nucleotide derivatives in the entorhinalhippocampal system: an autoradiographic study. Brain Res 1977;134:347-52.

${ }^{5}$ Sulakhe PV, Phillis JW. The release of $\left({ }^{3} \mathrm{H}\right)$ adenosine and its derivatives from cat sensorimotor cortex. Life Sci 1975;17:551-6.

${ }^{6}$ Sun M-C, McIlwain H, Pull I. The metabolism of adenine derivatives in different parts of the brain of the rat, and their release from hypothalamic preparations. $J$ Neurobiol 1976;7:109-22.

${ }^{7}$ Al-Khalidi UAS, Chaglassian TH. The species distribution of xanthine oxidase. Biochem J 1965;97: 316-20.

${ }^{8}$ Ågren H. Biological Markers in Major Depressive Disorders: A Clinical and Multivariate Study. Acta Universitatis Upsaliensis. Abstracts of Uppsala Dissertations from the Faculty of Medicine 405, 1981.

9 Åsberg M, Träskman L, Thorén P. 5HIAA in the cerebrospinal fluid: a biochemical suicide predictor? Arch Gen Psychiat 1976;33:1193-7.

${ }^{10}$ Endicott J, Spitzer RL. Use of the Research Diagnostic Criteria and the Schedule for Affective Disorders and Schizophrenia to Study Affective Disorders. Am J Psychiat 1979;136:52-6.

"Agren H. Symptom patterns in unipolar and bipolar depression correlating with monoamine metabolites in the cerebrospinal fluid: general patterns. Psychiatry Res 1980;3:211-23.

12 Andersen O, Johansson BB, Svennerholm L. Monoamine metabolites in successive samples of spinal fluid. Acta Neurol Scand 1981;63:247-54.

13 Andén N-E. Regulation of monoamine synthesis and utilisation by receptors. In: Szekeres L, ed. Handbook of Experimental Pharmacology. Springer Verlag, 1980:429-62.

${ }^{14}$ Lagercrantz H, Stjärne L. Evidence that most noradrenaline is stored without ATP in sympathetic large dense core nerve vesicles. Nature 1974;249: 843-4.

15 Israel M, Gautron J, Lesbats B. Fractionnement de l'agane electrique de la torpille: localisation de l'acetylcholine. J Neurochem 1970;17:1441-5.

${ }^{16}$ Whittaker VP. Subcellular localisation of neurotransmitters. Adv Cytopharmacol 1971;1:319-30.

${ }^{17}$ Zimmermann H, Penston CR. Adenosine triphosphate in cholinergic vesicles isolated from the electric organ of Electrophorus electricus. Brain Res 1976;11:365-76.

${ }^{18} \mathrm{Harms} \mathrm{HH}$, Wardeh G, Mulder AH. Effects of adenosine on depolarisation-induced release of various radiolabelled neurotransmitters from slices of rat corpus striatum. Neuropharmacology 1979;18:577-80.

19 Jhamandas K, Sawynok J. Methylxanthine antagonism of opiate and purine effects on the release of acetylcholine. In: Kosterlitz HW, ed. Opiates and Endogenous Opioid Peptides. Amsterdam: Elsevier North Holland Biomedical Press, 1976:161-8.

${ }^{20}$ Michaelis ML, Michaelis EK, Myers SL. Adenosine modulation of synaptosomal dopamine release. Life Sci 1979;24:2083-92.

${ }^{21}$ Winn HR, Rubio R, Berne RM. Brain adenosine production in the rat during 60 seconds of ischemia. Circulation Res 1979;45:486-92.

${ }^{22}$ Winn HR, Welsh JE, Rubio R, Berne RM. Brain adenosine production in rat during sustained alteration in systemic blood pressure. Am J Physiol 1980; 239:H636-41.

${ }^{23}$ Dascombe MJ, Milton AS. The effects of cyclic adenosine 3',5'-monophosphate and other adenine nucleotides on body temperature. $J$ Physiol (Lond) 1975;250:143-60.

${ }^{24}$ Feldberg W, Sherwood SL. Injections of drugs into the lateral ventricle of the cat. $J$ Physiol (Lond) 1954;123:148-67.

${ }^{25}$ Haulica I, Ababel L, Branistearm D, Topoliceanu F. Preliminary data on the possible hypnogenic role of adenosine. J Neurochem 1973;21:1019-20.

${ }^{26}$ Lewin E. Endogenously released adenine derivatives: a possible role in epileptogenesis. Trans Am Neurol Assoc 1976;101:192-4.

${ }^{27}$ Sweetman L. Urinary and cerebrospinal fluid oxypurine levels and allopurinol metabolism in the Lesch-Nyhan Syndrome. Fed Proc 1968;27,4:1055-9. 